\title{
Blood flow patterns in the human aorta studied by magnetic resonance
}

\author{
RICHARD H KLIPSTEIN, DAVID N FIRMIN, S RICHARD UNDERWOOD, \\ R SIMON O REES, DONALD B LONGMORE
}

From the Magnetic Resonance Unit, National Heart and Chest Hospitals, London

SUMMARY Magnetic resonance velocity mapping by the field even echo rephasing sequence was used to provide two dimensional velocity profiles in the ascending and the descending aorta. Flow patterns were studied in ten healthy volunteers by a display method that gave clear details of the profiles. Velocity profiles in the ascending aorta were skewed in systole with an axis of skew roughly symmetrical about the plane of the aortic arch. During diastole flow was reversed along the posterior left wall of the ascending aorta while it continued forwards at the anterior right wall. In the descending aorta plug flow occurred but with minimal skew. Flow along the right wall was reversed during diastole. Turbulent flow did not occur in the ascending or descending aorta of any healthy subject.

Magnetic resonance velocity mapping is a very powerful tool for the study of cardiovascular physiology. Its non-invasiveness, its quantitative two-dimensional data, its accuracy, and its high spatial resolution make it suitable for clinical use.

The complex phenomena of blood flow in the heart and blood vessels are worthy of study because they give an insight into the normal and pathological function of the cardiovascular system. Flow profiles are known to be modified in some diseases ${ }^{12}$ and are of crucial importance in others (for example steal syndromes). Our current knowledge of velocity profiles in the great vessels has come from invasive techniques such as catheter injection of oil droplets, ${ }^{3}$ and the hot-film anemometer, ${ }^{45}$ or from noninvasive ones such as the pulsed Doppler flow meter ${ }^{126}$ whose access to some blood vessels may be limited.

The recent advent of magnetic resonance velocity mapping ${ }^{78}$ using the field even echo rephasing sequence $^{9-11}$ has made it possible to obtain accurate velocity profiles in two dimensions at any point in the cardiac cycle. The technique is entirely noninvasive making it ideal for the study of flow patterns in vivo.

We report the analysis of aortic flow patterns in the ascending and descending aorta of 10 healthy volunteers.

Requests for reprints to Dr Richard H Klipstein, Magnetic Resonance Unit, National Heart and Chest Hospitals, 30 Britten Street, London SW3 6NN.

Accepted for publication 3 June 1987

\section{Principles of magnetic resonance velocity mapping}

The principles of the magnetic resonance phenomenon and the methods whereby these properties may be translated into non-invasive images are amply reviewed by Pykett et al. ${ }^{12}$ We will confine our description to those principles necessary to the understanding of how a parametric image of velocity may be produced. Although the phenomena follow the laws of quantum mechanics the quantum mechanical behaviour of populations of nuclei can accurately be described along the more readily comprehensible classical lines given below.

The hydrogen nucleus gives rise to the magnetic resonance signals used in clinical images. Nuclei spin on their axes, and in a magnetic field these axes of spin precess about the direction of the applied magnetic field (fig 1). The frequency of precession is directly proportional to the magnetic field that each nucleus experiences. The nuclei may be excited by the application of a radiofrequency pulse perpendicularly to the field and at the frequency of precession. This changes the angle (but not the axis) of precession by an amount depending on the strength and duration of the radiofrequency pulse. As the nuclei return to equilibrium they emit radio signals at the frequency of precession which can be detected in a 


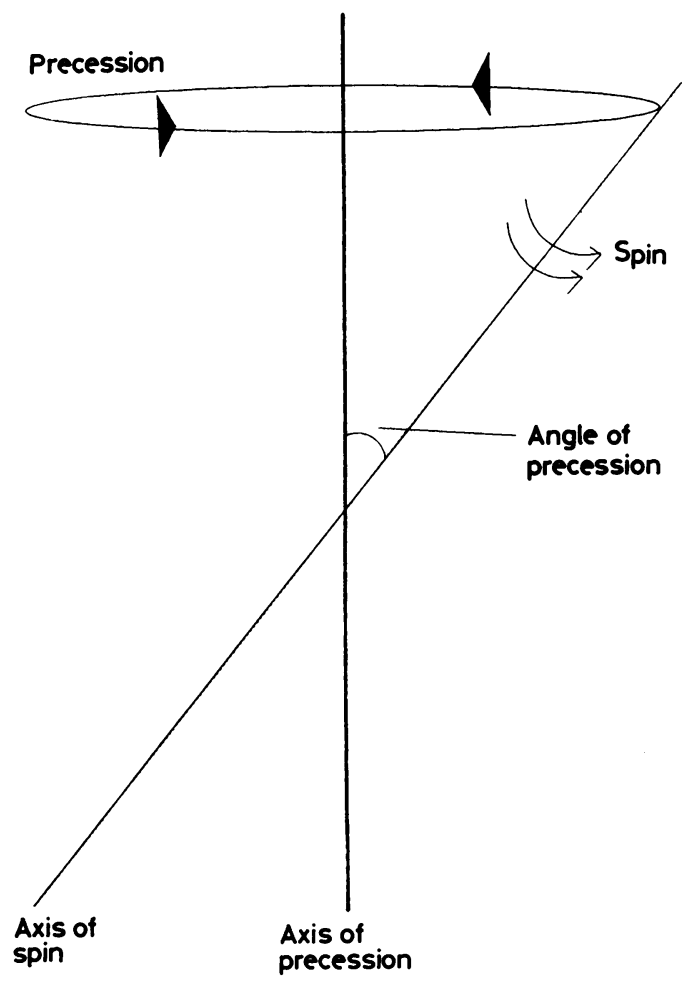

Fig 1 Definition of terms for the concepts of nuclear spin and precession of the axis of spin.

radio aerial and processed by a computer to produce the image.

The strength of the radio signal received at any time depends on the number of nuclei in phase. If the precessions are uniformly distributed around the circumference of the circle shown in fig 1 , no radio signal can be detected because those nuclei pointing in one direction have their signal cancelled by those diametrically opposite. If the precessions have a single phase, so that all point in one direction, a large radio signal is detectable.

The geometry of excitation with the radiofrequency pulse brings the nuclei into partial phase (for the hydrogen nucleus the nuclei occupy a little less than one third of the circumference of the circle). The radio signal decays away for several reasons; in the context of velocity mapping the most important of these is the loss of the phase coherence obtained during excitation.

The phase loss occurs because the magnetic field experienced by each nucleus is slightly different, either owing to its different chemical environment or to non-uniformities of the magnetic field or both. Because precession frequency is proportional to the field experienced by the nucleus, a range of precession frequencies is generated. As some nuclei precess more slowly and others more quickly their phase spreads around the circle.

Loss of phase can also be produced artificially by deliberately subjecting the sample to a non-uniform magnetic field. If the non-uniformity is generated by a gradient varying linearly from one side of the body to the other then phase will vary according to the position of the nucleus in space along the gradient. This property is used to generate the spatial resolution, which in conjunction with computerised image reconstruction methods ${ }^{13-15}$ produces images.

If phase has been disturbed by applying a gradient for a time, it may be restored by applying an equal and opposite gradient for an identical length of time. Nuclei whose precession was speeded up are now slowed down and vice versa. The precession of the nuclei returns to phase coherence, a phenomenon known as gradient refocusing.

The equations used by the image reconstruction method of two dimensional Fourier transformation $^{15}$ may be solved for the phase of the nuclei in any voxel. In magnetic resonance studies a slice of finite thickness is imaged, so that the image element has a height equal to the slice thickness and cross section equal to the pixel area. This three dimensional image element is called a voxel. An image in which each voxel has the value of the average phase within it is called a phase image.

We can encode velocity as phase by superimposing gradients on those used for imaging. If a gradient is put on in the direction in which velocity is to be measured (either perpendicular to the slice or in any direction within it), the nuclei in each voxel will receive a phase signature. If an equal and opposite gradient is then put on in this direction this phase signature will be lost by cancellation of the phase disturbance imposed by the first gradient.

Cancellation will only occur if the nucleus remains in the same place in the gradient. If it is moving up the gradient, its first phase signature will be more than cancelled by the second, since by the time the second is applied the nuclei are experiencing a stronger magnetic field. A negative phase will result. If it is moving down the gradient a positive phase will result. This resultant phase will be proportional to the velocity component in the direction of the gradient.

For very high velocities, the over or under cancellation may be so great that the resultant phase differs by more than one revolution. Because phase is an angle, it may only uniquely lie within the range of a single revolution. Two velocities, one producing a 
given phase angle and the other a phase angle equal to one or more revolutions plus the first are interpreted as being identical. The size and duration of the velocity encoding gradients and the time between them are thus chosen so that the maximum velocity encountered will give a phase angle which is less than one complete revolution. Since with bidirectional flow it is often necessary to consider high velocities in both directions, the maximum in one direction must not exceed half a revolution. (If it is known before acquisition that maximum forward velocity will be larger than maximum reverse velocity, the gradients can be tailored so that the sum of phase angles from the maximum forward and reverse velocities is one complete revolution.)

In practice, inhomogeneities of the main magnetic field produce artefacts on the phase map. This problem is overcome by acquiring two images, one with velocity encoding gradients and the other without, and subtracting the two phase maps.

A further refinement is necessary before velocity mapping is practical in large vessels. Where accelerations are large, a voxel will contain a wide range of different velocities, and therefore a large number of resultant phases after the flow encoding sequence. Because these are out of phase with each other there is signal loss. This problem is overcome by even echo rephasing which refocuses the range of phases in a manner similar to gradient refocusing. ${ }^{9}$ Even so, gross turbulence within a voxel will still result in signal loss.

\section{Patients and methods}

A Picker International Vista 2055 magnetic resonance machine, operating at $0.5 \mathrm{~T}$ was used. Ten healthy volunteers without a history of cardiac disease were studied after they had given informed consent. Electrocardiographically gated velocity maps were obtained in the transverse plane by the field even echo rephasing sequence with gradient modification to encode velocity in the phase of the magnetic resonance signal (see principles of magnetic resonance velocity mapping above). To compensate for inhomogeneities of the magnetic field, a reference image without gradient modification was subtracted from the one with modification. Up to 12 frames (depending on heart rate) were obtained at a level at which the ascending aorta was perpendicular to the transverse plane-that is the level of the right pulmonary artery. Views of both the ascending and the descending aorta were thus obtained in a single transverse section. The first frame corresponded to a point in the cycle $20 \mathrm{~ms}$ after the onset of the electrocardiographic $\mathbf{R}$ wave; frames were obtained at $50 \mathrm{~ms}$ intervals.
A $128 \times 128$ pixel image was sampled twice (improving the signal to noise ratio). Acquisition thus took about five minutes depending on heart rate. The field of view was $45 \mathrm{~cm}$ but the image was interpolated in two dimensions during reconstruction, giving a voxel dimension of $1.8 \times 1.8 \times 10 \mathrm{~mm}$. Two dimensional Fourier transformation in which the magnitude and phase were calculated for each pixel was used as the method of image reconstruction. Masking of the phase reconstruction to show zero veolocity in pixels with a magnitude value of less than $10 \%$ of maximum reduced the random phase values caused by background noise.

After acquisition, the velocity maps were transferred to an off line computer system (Sperry Univac V77-600) for further processing. A window of $32 \times$ 32 pixels was selected and positioned such that the ascending aorta appeared centrally in the $220 \mathrm{~ms}$ frame. This window position was used for all images in the cycle, thus ensuring accurate registration of frames for cine display. These images were scaled so grey levels corresponded exactly on all of them. They were then stored and displayed with a coarse grey scale of 12 levels to produce a pseudocontouring effect. ${ }^{16}$ An identical process was used for descending aortic windows over the same velocity maps.

\section{Results}

Figure 2 shows a series of contoured velocity profiles at the indicated points of the cardiac cycle in the ascending aorta of a typical subject. Mid-grey signifies no flow, black flow out of the image plane, and white flow into the image plane. All the subjects studied showed qualitatively identical patterns throughout the cycle with the exception of one in whom the only difference was a peak diastolic reverse flow which was rotated towards the left side of the aorta by $90^{\circ}$.

Ascending aortic flow maps showed the following features: (a) plug flow occurred during most of systole. (Plug flow is the smooth flow of an inviscid fluid with a velocity profile which is uniform across the vessel. Instead of flow being slow near the edges of the pipe and rapid in the middle, a quasi-solid "plug" of fluid moves down the pipe.) The velocity profile was skewed towards the posterior left wall of the vessel; $(b)$ during diastole, flow continued rapidly forward at the anterior right wall while simultaneously moving rapidly backwards at the posterior left wall; (c) flow never became turbulent. In fig 3 the data of fig 2 are redisplayed as a perspective three dimensional plot. This eases interpretation, but it requires considerable image processing. The spatial localisation of peak velocities (mean (SD)) in the 


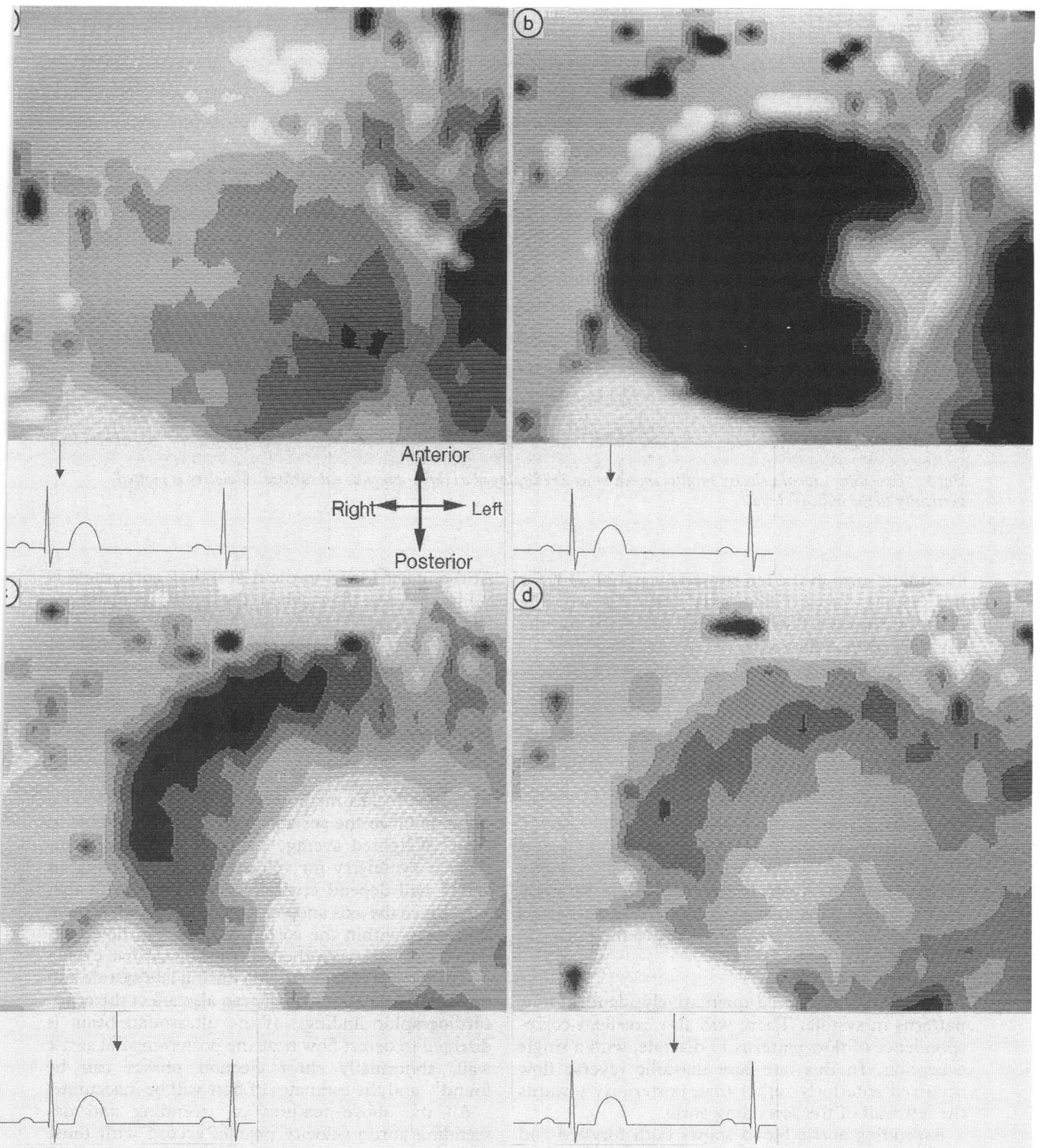

Fig 2 A series of pseudocontoured velocity maps of the ascending aorta in a healthy volunteer. Each grey level corresponds to a range of flow velocities. Mid-grey signifies no flow, black flow out of the image plane, and white flow into the image plane. The times for each frame are indicated by the accompanying electrocardiogram trace, and the orientation is as shown. Note the skewing of the velocity during rapid ejection (a), the start of flow reversal (b) at $220 \mathrm{~ms}$ (well before aortic valve closure), the dominance of the reverse flow channel while forward flow continues at the anterolateral wall at $370 \mathrm{~ms}$ (c), and the cessation of reverse flow at $570 \mathrm{~ms}(d)$. Note that all the velocities in (d) are either zero or in the forward direction. 

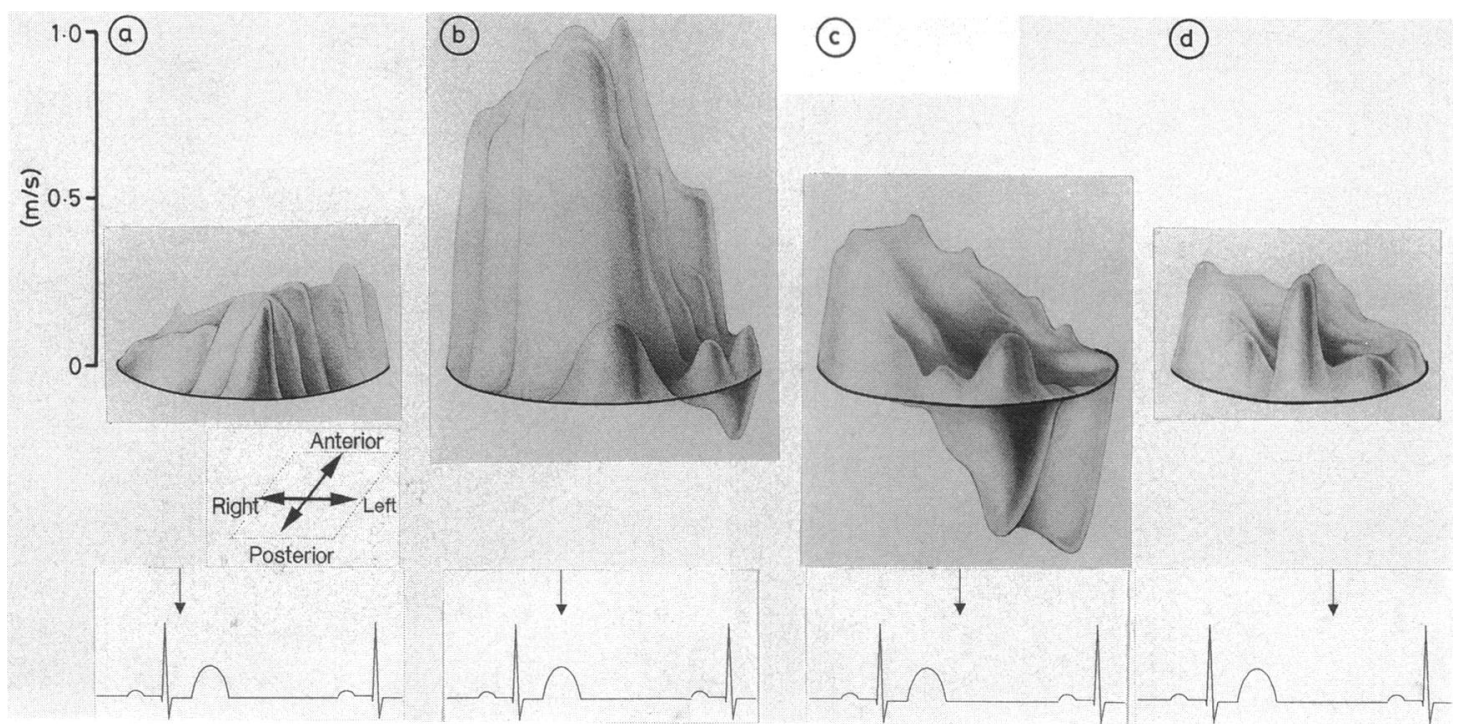

Fig 3 Two-dimensional velocity profiles shown in fig 2 redisplayed as three-dimensional surfaces. Velocity is plotted vertically to the indicated scale.

ascending aorta in the ten subjects was $129^{\circ}(14)$ for the systolic forward flow axis $-89^{\circ}$ (20) for the diastolic forward flow axis, and $122^{\circ}$ (15) for the diastolic reverse flow axis.

The descending aortic flow maps were broadly similar to those of the ascending aorta. The important differences were that: (a) plug flow occurred, but the velocity profile has lost its skewness at this level; (b) flow reversed at the right wall of the vessel during diastole in all but two subjects.

\section{Discussion}

The ability to study two-dimensional velocity profiles non-invasively makes it possible to study complex flow phenomena without the disturbances that may be induced by more invasive methods.

\section{FLOW PATTERNS}

All the subjects showed qualitatively identical flow patterns in systole. There was also excellent correspondence of flow patterns in diastole, with a single exception. In this one case diastolic reverse flow occurred anteriorly rather than posteriorly towards the left wall of the ascending aorta.

Ascending aortic blood shows both forward and reverse flow during diastole. The total reverse flow is too great for all of it to pass into the coronary ostia; rather it seems that a circulation is set up around the sinus of Valsalva, with a proportion of the recirculating blood flowing into the coronary arteries.
At the imaged level in the descending aorta there is a little reverse flow when the pressure upstream falls. In two subjects, reverse flow in the descending aorta was not seen, implying that the leading edge of the recirculating flow occurred near the imaged plane (that is high in the descending aorta).

The skewness of the velocity profile and its axis of symmetry (which lies in the plane of the aortic arch) has implications for the accuracy of aortic blood flow as calculated by range gated Doppler ultrasound. Skewness makes methods of interpreting flow at a single point in the aorta as mean flow vulnerable to error. Wéighted average methods assuming semicircular symmetry for velocities obtained along an axis $^{16}$ will depend critically on the choice of that axis. Since the axis and velocity peaks do not remain stationary within the aorta, the errors in flow estimates will vary with the point in the cardiac cycle.

The reverse flow occurring during late systole and throughout most of diastole can also affect the echocardiographic findings. If the ultrasound beam is directed to detect flow near the posteromedial aortic wall, abnormally short ejection phases can be found ${ }^{17}$ and the estimates of flow will be inaccurate.

All the above features of ascending and descending aortic velocity profiles accord with those found in animals by invasive methods, such as oil droplet injection, ${ }^{3}$ and hot-film anemometry, ${ }^{4518}$ and with ultrasound findings. ${ }^{19}$ They also accord with ultrasound work in man. ${ }^{1} 26$ The restriction of axes to anteroposterior and right-left in some 
anemometer ${ }^{4}$ and ultrasound ${ }^{19}$ work masks the true axis of skew as revealed by the two-dimensional magnetic resonance velocity maps.

The flow patterns obtained resemble those obtained by Paulsen and Hasenkam in the ascending aorta in dogs $5 \mathrm{~cm}$ above the aortic valve. ${ }^{5}$ They showed that the two-dimensional velocity profiles changed considerably from a level $2 \mathrm{~cm}$ above the valve to a level $5 \mathrm{~cm}$ above it. Thus when studies are compared the levels must correspond. The reference plane in this study is that of the right pulmonary artery, which is a little more than $5 \mathrm{~cm}$ from the centre of the aortic valve. ${ }^{20}$

The phenomena occurring as blood is ejected into the aorta are complex. The viscous properties of blood, the pulsatility of the flow, the elasticity of the aorta, the propagation and reflection of the shock waves generated during ventricular contraction, the entrance region effects, and flow into vessels arising from the aorta must all be taken into account ${ }^{21}$ when theories are proposed to account for the observed phenomena.

One consistent finding in this and the other studies $^{22}$ has been skewing of the systolic velocity profile towards the posterior wall of the ascending aorta during systole. The velocities are fastest where the radius of curvature is least. This finding disagrees with the theoretical predictions of McDonald. ${ }^{23}$ Seed and Wood have provided a simple model of flow of an inviscid fluid in a curved pipe ${ }^{4}$ which suggests that velocities are inversely proportional to the radius of curvature-that is faster velocities occur near the wall on the inside of the curve. In our study the gradient of the systolic profile reached a maximum in the plane of the aortic arch, which is consistent with the theory of Seed and Wood.

In these normal subjects, there was no turbulent flow. Anything more than minimal turbulence would show as signal loss not correctable by even echo rephasing. Some have reported turbulence, ${ }^{524}$ particularly when anemometry has been used in smaller animals, or they have surmised that it occurs. ${ }^{25}$ This may be because the presence of the anemometer probe in the aortic lumen disturbs flow that is already at the limits of its stability as nonturbulent flow. Some have commented on signs of instability or streamlines stopping short of fully developed turbulence because of the short duration of very rapid flow. ${ }^{5}$

Simultaneous forward and reverse flow can occur in a single channel in a vessel without causing turbulence, provided that the shearing forces are not too great and no other generator of turbulence, such as a stenotic valve, is present. This may be appreciated by considering ripples on the surface of water.
At any instant there are peaks and troughs. If a velocity map were to be acquired in the plane of the surface of the water, recording velocities into and out of the plane, there would be some areas of upward velocity and adjacent areas of downward velocity, bounded by lines of zero velocity. Turbulent flow is not occurring in these ripples even though bidirectional flow would be seen on the velocity map.

\section{MAGNETIC RESONANCE VELOCITY MAPPING}

Magnetic resonance velocity mapping has great advantages over other methods for the study of flow patterns. Oil droplet injection and hot film anemometry are invasive and time-consuming. They are not suited to routine clinical use. By use of a special mounting apparatus, ${ }^{5}$ hot film anemometry can be adapted to the measurement of twodimensional velocity profiles in animals. Its technical limitations make it unsuitable for the study of the boundary layer.

Pulsed Doppler echocardiography can be used to provide velocity profiles in large vessels in both animals and in man. It has the advantage of being noninvasive, but its velocity profiles must be one-dimensional if a sufficient number of velocity levels are to be resolved. It is possible to obtain twodimensional echocardiographic velocity profiles ${ }^{26}$ but with only a few steps of velocity, ${ }^{27}$ which is insufficient for the generation of enough flow contour levels. Pulsed Doppler methods also suffer from aliasing at high velocities. ${ }^{28}$ Movement of the aortic wall into and out of the sample volume makes it very difficult to study the boundary layer, though tracking methods may partially eliminate artefact from this cause.

Magnetic resonance velocity mapping has the advantages of being non-invasive, intrinsically twodimensional, and has very high resolution of velocity. Comparisons of cardiac output derived from chamber volume estimates ${ }^{29}$ and those measured by summation of aortic velocities in all frames show agreement to within $7 \%$. The use of the field even echo rephasing sequence to avoid signal loss permits its use in both the ascending and the descending aorta as well as in other vessels of clinical importance. Up to 12 frames per cycle may be produced in short acquisition times and access to some vessels is not limited as it is with echocardiography. Because it does not depend on range gating, it is not limited by movement of the aortic wall from frame to frame, making it suitable in principle for the study of the boundary layer.

Although the present study used a resolution (before interpolation) giving a pixel size greater than the size of the boundary layer, higher resolution is 
not difficult to achieve. The close-packed contours of fig 2 confirm what has been suspected from hot film anemometry-that is that the boundary layer is thin $(1-2 \mathrm{~mm})$, and that shear rates in it are extremely high.

Velocity mapping is suitable for use in small vessels where the lower accelerations make velocity easier to measure. Maps have been obtained in carotid arteries, vertebral arteries, and jugular veins, ${ }^{7}$ and in femoral arteries and veins. ${ }^{30}$

\section{DISPLAY SYSTEM}

The use of a pseudocontoured display ${ }^{16}$ has advantages over the use of contour lines, especially when cine displays are used. This is because the effect of having far fewer grey scale levels than pixel values forces whole ranges of pixel values to be displayed as a single shade of grey. Each shade of grey thus signifies a specific velocity range.

The use of a restricted number of grey levels does not overload the visual system, thereby simplifying interpretation. By adjustment of window width and level (analogous to contrast and brightness), the contours can be made to move over the velocity profile giving considerable information about its threedimensional structure. With training it is possible to visualise this structure very clearly and quickly.

As well as its information content, this type of display does not require image processing. It is produced very simply and rapidly by switching the display monitor to a coarse grey scale. While the quasi-three-dimensional displays of fig 3 may be easier to comprehend without training, their routine production requires considerable computing power and time.

\section{Conclusion}

Magnetic resonance velocity mapping is a noninvasive method that provides accurate twodimensional velocity maps anywhere in the cardiovascular system. Its application to the ascending and descending aorta clearly defines consistent normal flow patterns and the nonturbulent nature of the flow. Its application to the study of the boundary layer, and to the modification of flow patterns in the presence of atheroma in smaller vessels will be of help both in diagnosis and in contributing to our understanding of the pathophysiology of cardiovascular disease.

We thank the Board of Governors of the National Heart and Chest Hospitals, the Coronary Artery Disease Research Association (CORDA), and Picker International Ltd. We thank Mr G L Nayler of Picker International Ltd for his technical help in obtaining the velocity maps.

\section{References}

1 Jenni R, Vieli A, Anliker M, Krayenbuehl HP. Doppler-echocardiography: its advantages and limitations in adult cardiology. In: Roelandt $D$, ed. The practice of $M$-mode and two dimensional echocardiography. The Hague: Martinus Nijhoff, 1983;90-7.

2 Jenni R, Ruffman K, Vieli A, Anliker M, Krayenbuehl HP. Dynamics of aortic flow in hypertrophic cardiomyopathy. Eur Heart $J$ 1985;6:391-8.

3 Ohlsson NM. Left heart and aortic flow patterns in the dog. Precision motion analysis of high speed (270 frames/sec.) cinefluorographic recordings. Acta Radiol 1962;suppl 213:1-80.

4 Seed WA, Wood NB. Velocity patterns in the aorta. Cardiovasc Res 1971;5:319-30.

5 Paulsen PK, Hasenkam JM. Three-dimensional visualisation of velocity profiles in the ascending aorta in dogs measured with a hot film anemometer. $J$ Biomechan 1983;16:201-10.

6 Jenni R, Vieli A, Ruffman K, Krayenbuehl HP. A comparison between single gate and multigate ultrasonic Doppler measurements for the assessment of the velocity pattern in the human ascending aorta. Eur Heart J 1984;5:948-53.

7 Bryant DJ, Payne JA, Firmin DN, Longmore DB. Measurement of flow with NMR imaging using a gradient pulse and phase difference technique. $J$ Comput Assist Tomogr 1984;8:588-93.

8 Underwood SR, Firmin DN, Klipstein RH, Rees RSO, Longmore DB. Magnetic resonance velocity mapping: clinical application of a new technique. $\mathrm{Br}$ Heart $J$ 1987;57:404-12

9 Pattany PM, Nayler GL. High velocity flow imaging by even echo rephasing. Society of Magnetic Resonance in Medicine, Fourth Annual Meeting, London, 1985. Book of Abstracts, 599-600. Berkeley, California: Society of Magnetic Resonance in Medicine.

10 Longmore DB, Firmin DN, Nayler GL, Underwood SR, Klipstein RH. Cine magnetic resonance blood flow imaging in clinical use [Abstract]. Mag Reson Imaging 1986;4:157.

11 Nayler GL, Firmin DN, Longmore DB. Blood flow imaging by cine magnetic resonance. J Comput Assist Tomogr 1986;10:715-22.

12 Pykett IL, Newhouse JH, Buonanno FD, et al. Principles of nuclear magnetic resonance imaging. Radiology 1982;143:157-68.

13 Hounsfield GN. Computerised transverse axial scanning (tomography), Part I. Description of system. $\mathrm{Br}$ J Radiol 1973;46:1016-22.

14 Lauterbur PC. Image formation by induced local interactions. Examples employing nuclear magnetic resonance. Nature 1973;242:190-1.

15 Kumar A, Welti D, Ernst R. NMR Fourier zeugmatography. J Magn Reson 1975;18:69-83.

16 Delp EJ, Buds AJ. Digital image processing. In: Buda AJ, Delp EJ, eds. Digital cardiac imaging. Dordrecht: Martinus Nijhoff, 1985:5-23.

17 Louie EK, Maron BJ, Green KJ. Variations in flow-velocity waveforms obtained by pulsed Doppler 
echocardiography in the normal human aorta. Am J Cardiol 1986;58:821-6.

18 Freis ED, Heath WC. Hydrodynamics of aortic blood flow. Circ Res 1964;14:105-16.

19 Peronneau P, Sandman W, Xhaard M. Blood flow patterns in large arteries. Ultrasound in Medicine 1977;38:1193-208.

20 Gray H. Gray's anatomy. 35th ed. Warwick R, Williams PL, eds. London: Longman, 1973:617.

21 McDonald DA. Blood flow in arteries. 2nd ed. London: Edward Arnold, 1974.

22 Caro CG, Pedley TJ, Schroter RC, Seed WA. The mechanics of the circulation. Oxford: Oxford University Press, 1978:316-7.

23 McDonald DA. Blood flow in arteries. 2nd ed. London: Edward Arnold, 1974:90.

24 McDonald DA. Blood flow in arteries. 2nd ed. London: Edward Arnold, 1974:80.

25 Caro CG, Pedley TJ, Schroter RC, Seed WA. The mechanics of the circulation. Oxford: Oxford
University Press, 1978:332-3.

26 Miyatake K, Mitsunori O, Kinoshita N, et al. Clinical applications of a new type of real-time two dimensional Doppler flow imaging system. Am J Cardiol 1984;54:857-68.

27 Miyatake K, Izumi S, Shimizu A, et al. Right atrial flow topography in healthy subjects studied with real-time two dimensional Doppler flow imaging technique. J Am Coll Cardiol 1986;7:425-31.

28 Snider AR. Doppler. echocardiography: basic principles and clinical applications. In: Buda AJ, Delp EJ, eds. Digital cardiac imaging. Dordrecht: Martinus Nijhoff, 1985:182-206.

29 Longmore DB, Klipstein RH, Underwood SR, et al. Dimensional accuracy of magnetic resonance studies of the heart. Lancet 1985;i:1360-2.

30 Underwood SR, Firmin DN, Klipstein RH, Rees RSO, Longmore DB. Magnetic resonance velocity mapping:clinical application of a new technique. $\mathrm{Br}$ Heart J 1987;57:404-12. 